Recepción: 20 / 04 / 2017

Aceptación: 20 / 05 / 2017

Publicación: 15 / 07 / 2017

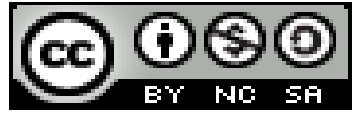

Ciencias económicas y empresariales Artículo de investigación

\title{
Gestión estratégica para la administración financiera de negocios, en productos y servicios en el sector de pequeñas y medianas empresas
}

Strategic management for the financial administration of businesses, in products and services in the sector of small and medium enterprises

Gestão estratégica para a gestão financeira de negócios, produtos e serviços no sector das pequenas e médias empresas

Julio C. Pacheco-Jara ${ }^{\mathrm{I}}$ julio.pachecoj@ug.edu.ec

Leonor M. Morales-Gallegos II leonor.moralesg@ug.edu.ec

Marlín M. Alvarado-Zuñiga III marlin.alvaradoz@ug.edu.ec

Correspondencia: julio.pachecoj@ug.edu.ec 


\section{Resumen}

La intención del presente trabajo es realizar un análisis sobre la importancia de contar con un sistema de contabilidad actualizado para controlar las actividades productivas de manera adecuada, así como contar con un correcto manejo de los recursos y programas de macroeconomía. El sistema de Contabilidad de Costos por Absorción, el cual se utiliza en el Ecuador es menos eficiente que los modelos de costeo estándar o variable, las PYMES del Cantón Guayaquil muestran fortalezas como: infraestructura productiva en inversiones, experiencia empresarial, conocimiento del mercado; y sus debilidades son: el limitado conocimiento sobre el manejo financiero estratégico, deficiente manejo presupuestario y débil gestión en la determinación de costos reales. Por esto se plantea un Modelo de Gestión Estratégica Gerencial para corregir los deficientes mecanismos de manejo financiero, que son la causa principal de la insuficiencia de la Administración Financiera en las PYMES; este modelo de gestión estratégica consta de cuatro componentes: un modelo de Administración Financiera de Negocios de una PYME, Resultados Económicos de las Empresas, Gestión Financiera en la Rentabilidad Sostenible, Estado de Proforma. De forma general una PYME tiene una estructura de inversiones cuya rentabilidad económica y financiera debe determinarse y permitir una evaluación periódica y continua para la gerencia general; por tanto este Modelo de Gestión Estratégica Gerencial permitirá mejorar el nivel de productividad y competitividad en las ofertas de los productos o servicios de cada PYME.

Palabras Claves: Contabilidad; macroeconomía; financiero; estratégico; mercado. 


\section{Summary}

The purpose of this paper is to analyze the importance of having an updated accounting system to control productive activities in an adequate manner, as well as to have a correct management of macroeconomic resources and programs. The Absorption Cost Accounting system, which is used in Ecuador is less efficient than standard or variable costing models, Guayaquil Canton SMEs show strengths such as: productive infrastructure in investments, business experience, market knowledge; And its weaknesses are: limited knowledge about strategic financial management, poor budget management and weak management in determining real costs. For this reason, a Management Strategic Management Model is proposed to correct the poor financial management mechanisms, which are the main cause of the insufficiency of the Financial Administration in SMEs; This strategic management model consists of four components: a Business Financial Management model of an SME, Business Economic Results, Financial Management in Sustainable Profitability, Proforma State. Generally an SME has an investment structure whose economic and financial profitability must be determined and allow a regular and continuous evaluation for general management; Therefore, this Management Strategic Management Model will allow to improve the level of productivity and competitiveness in the offerings of the products or services of each SME.

Key Words: Accounting; macroeconomics; financial; strategic; market 


\section{Resumo}

A intenção deste trabalho é analisar a importância de ter um sistema de contabilidade atualizada para controlar as atividades de produção de forma adequada e ter uma gestão adequada dos recursos e programas de macroeconomia. O sistema de contabilidade analítica para a absorção, que é usado no Equador é menos eficiente do que os modelos padrão ou custeio variável, Canton Guayaquil PME mostram pontos fortes como investimentos em infraestrutura produtiva, experiência empresarial, conhecimento de mercado; e pontos fracos são o conhecimento limitado sobre a gestão financeira estratégica, má gestão financeira e gestão fraca na determinação dos custos reais. Portanto, a Gestão Estratégica Model Management é proposto para corrigir mecanismos de gestão financeira pobres, que são a principal causa da gestão financeira inadequada nas PME; Este modelo de gestão estratégica consiste em quatro componentes: um modelo de gestão financeira das PME negócios, resultados econômicos da empresa, rentabilidade sustentável Gestão Financeira, Estado do Proforma. SME geralmente tem uma estrutura de investimentos cujos econômico e rentabilidade financeira ser determinada e permitir a avaliação regular e contínua para a administração geral; de modo que este modelo de gestão estratégica irá melhorar o nível de produtividade e competitividade nas ofertas de produtos ou serviços cada PME.

Palavras-chave: Contabilidade; macroeconomia; finanças; mercado estratégico. 


\section{Introduccion.}

En la actualidad, en el Ecuador se utiliza un sistema de contabilidad convencional, denominado sistema contable por Absorción, que presenta muchas insuficiencias en la determinación del costo real del producto o servicio, razón por la cual en los países de mayor desarrollo ha sido sustituido por otros sistemas de mejor efectividad empresarial, manejo financiero y tributario gubernamental. En ningún país de economía de primer mundo se está aplicando la contabilidad por Absorción, porque en lugar de beneficiar perjudica el desenvolvimiento de las actividades económicas de una nación.

El modelo de gestión basado en los costos estándar o variable, es mucho más eficiente que los modelos de costeo tradicional o absorción, porque evita o elimina el despilfarro de recursos y establece un verdadero sistema de gestión estratégica en el proceso gerencial de la empresa.

En la actualidad el Sector Empresarial del cantón Guayaquil, se encuentra buscando mecanismos de apoyo financiero para salir de la crisis económica y de competitividad, originada por las ofertas de menor precio de las empresas extranjeras de alcance global. Esto se expresa en los mayores precios monetarios de los productos y servicios que ofertan las empresas locales, en relación a las ofertas de las empresas globales que comercializan productos en el Ecuador, y especialmente en el cantón Guayaquil.

Las empresas globales, en general realizan una gestión empresarial utilizando un modelo de costeo basado en la administración de actividades y las empresas guayaquileñas aplican un sistema de costeo a los productos y servicios, lo cual son diferencias, que marcan ventajas competitivas evidentes que favorecen a las empresas globales. 
De las investigaciones realizadas sobre los aspectos incidentes de la crisis de los procesos gerenciales de la empresa, se ha podido identificar que las empresas globales, manejan mucho mejor el sistema de información en el proceso de costos, calidad y precios, en la gestión de ofertas de productos y servicios, que las empresas guayaquileñas.

\section{Materiales y métodos (Metodología)}

Gestión Estratégica Gerencial para la Administración Financiera de Negocios, en Productos y Servicios en el Sector de la Pequeña y Mediana Empresa del cantón Guayaquil, la modalidad de la investigación es, Descriptiva y Aplicada.

\section{Tipo de Investigación}

El tipo de investigación se considera de campo y bibliográfica, por las siguientes características:

a) Por los objetivos.-Es Investigación Aplicada, porque toma los conocimientos de la Investigación Bibliográfica y permite encontrar soluciones al problema planteado

b) Por el alcance.- Es Investigación Descriptiva, porque describe la forma de cómo se establece la organización de un Modelo de Gestión Estratégica Gerencial para la Administración Financiera de Negocios, en Productos y Servicios en el Sector de Pequeña y Mediana Empresa del cantón Guayaquil.

c) Por el lugar.- Es Investigación de Campo, porque se recopilaron los datos en el mismo lugar donde producen los hechos problemáticos. 


\section{Universo y muestra}

Esta investigación está limitada por un universo que se compone de 60000 personas relacionadas con el problema, 10000 en el Sector Industrial, 32000 en el Sector Comercial, 8000 Profesionales, 1000 en el Sector Gobierno, 2200 Académicos y 6000 en el Sectores de PYMES, que realizan actividades empresariales por cuenta propia, en la ciudad de Guayaquil Provincia del Guayas. Es sobre este universo donde se aplicaron las encuestas a 398 de los involucrados, los que fueron seleccionados por el sistema aleatorio simple, como Muestra representativa del Universo en estudio.

\section{Técnicas e instrumentos}

\section{1) Técnica de recopilación bibliográfica}

El proceso bibliográfico de estudio se realizó por medio de la utilización de la información de manera sistemática de las fuentes secundarias de: libros, boletines, folletines, y del banco de datos del Internet sobre el tema en estudio.

\section{2) Técnicas de recopilación de campo}

Las técnicas en la recopilación de campo, fueron las siguientes: Encuestas, Observación Directa, Entrevista.

\section{Métodos}

Método científico-Por medio de este método se pudo formular interrogantes sobre la realidad, con base en la teoría ya existente en los fundamentos científicos de los autores de los libros, tratando de hallar soluciones a los problemas planteado. 
Método heurístico.-Se fundamenta en el proceso inductivo, lo que permite investigar el problema y formular hipótesis.

Observacion situacional.-El proceso de investigación se inició con la recopilación de información sobre la situación de insuficiencia en la aplicación de la aplicación del costeo en los productos y servicios, mediante el Proceso Gerencial de cada empresa del cantón Guayaquil.

Experimentación.-En este paso se procedió a la verificación del universo de personas involucradas en el problema formulado, con la aplicación de las técnicas del censo poblacional, con el cual se debería trabajar en el proceso investigativo.

Comparación y generalización-Posteriormente se estableció los resultados de la exploración experimental logrando una generalización y conceptualización del problema, en los aspectos informativos más importantes, y con estos resultados establecer un plan de acciones para lograr obtener todos los objetivos planteados.

Método analítico- sintético.- Este método de investigación, permite examinar y resumir los problemas existentes en el campo donde se realiza la investigación. 


\section{Resultados.}

Imagen 1. Tendencia General de la Encuesta

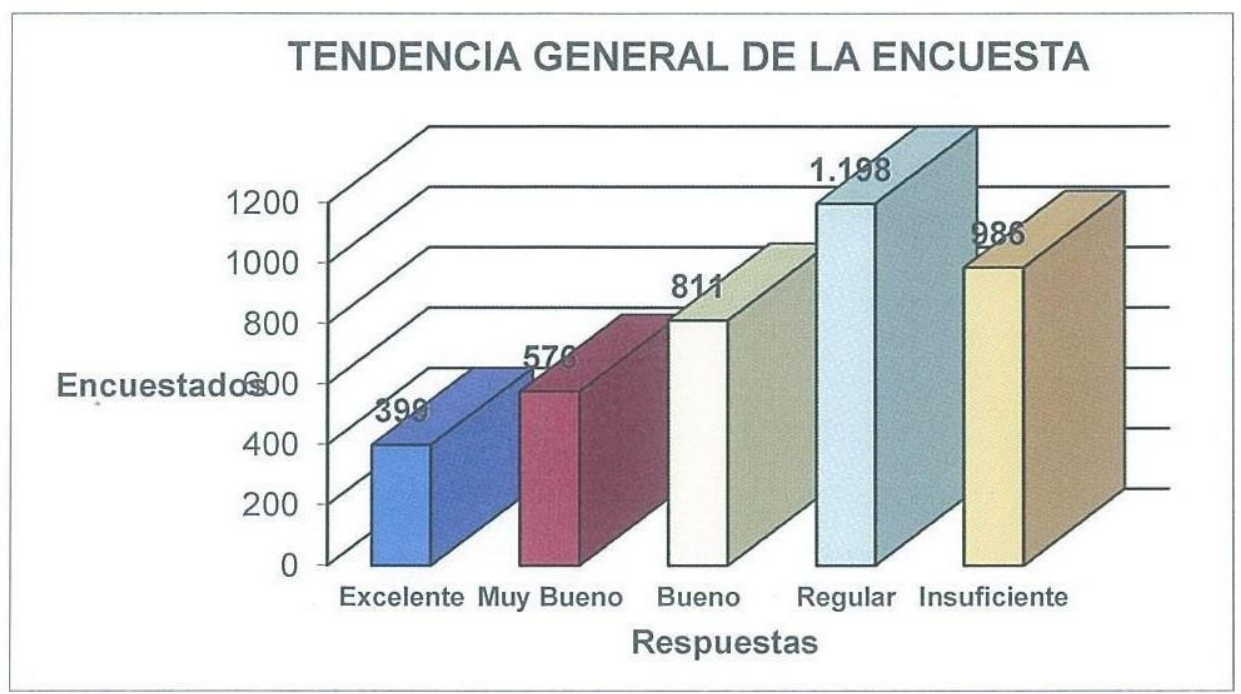

Fuente: Elaboración Propia.

Imagen 2. Porcentaje General de la Encuesta

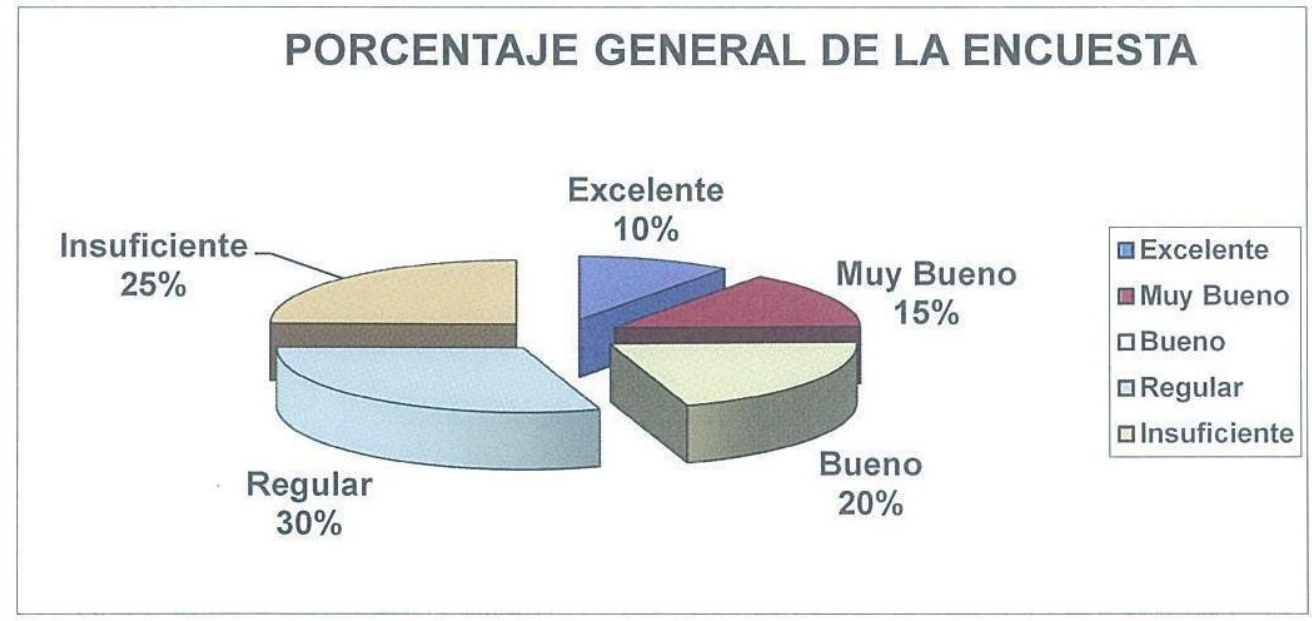

Fuente: Elaboración Propia.

Los deficientes mecanismos de manejo financiero, son la causa principal en la insuficiencia de la Administración Financiera de Negocios, en Productos y Servicios en el Sector de Pequeña y Mediana Empresa del cantón de Guayaquil, debido a una inadecuada Gestión Estratégica Gerencial. 


\section{Conclusiones.}

Del desarrollo del proceso investigativo y de los contenidos de los componentes del Modelo de Gestión de Estratégica Gerencial para la Administración Financiera de negocios, en Productos y Servicios en el Sector de Pequeña y Mediana Empresa del cantón Guayaquil, se deducen las siguientes conclusiones:

1) Los contenidos desarrollados en Modelo de Gestión Estratégica Gerencial mejorará la Administración Financiera de Negocios, en Productos y Servicios en el Sector de Pequeña y Mediana Empresa del cantón Guayaquil, son i mecanismos adecuados para ser aplicados por los niveles gerenciales y permitirán mejorar el nivel de productividad y competitividad en las ofertas de los productos o servicios de cada PYME

2) Los contenidos están diseñados para que sean aplicados en cualquier PYME que deseen implementar el Modelo de Gestión Estratégica Gerencial mejorará la Administración Financiera de Negocios, en Productos y Servicios en el Sector de Pequeña y Mediana Empresa del cantón Guayaquil.

3) Que el Modelo de Gestión Estratégica Gerencial es una variable independiente de cambio, que puede ayudar a mejorar la Administración Financiera de Negocios, tiene como finalidad aportar al mejoramiento de los niveles de productividad y competitividad en las ofertas de Productos y Servicios en el Sector de Pequeña y Mediana Empresa del cantón Guayaquil.

\section{Recomendaciones.}

Del desarrollo del proceso investigativo y de los contenidos de los componentes del Modelo de Gestión Estratégica Gerencial mejorará la Administración Financiera de Negocios, en Productos y 
Servicios en el Sector de Pequeña y Mediana Empresa del cantón Guayaquil, se deducen las siguientes recomendaciones:

1) Los empresarios y gerentes del Sector de la Pequeña y Mediana Empresa del cantón Guayaquil, apliquen el Modelo de Gestión Estratégica Gerencial para la Administración Financiera de Negocios, porque tiene por finalidad el mejoramiento en el nivel de productividad y competitividad de dicho sector.

2) La implementación del Modelo de Gestión Estratégica Gerencial para la Administración Financiera de Negocios, en Productos y Servicios en el Sector de Pequeña y Mediana Empresa del cantón Guayaquil, los empresarios y gerentes pueden hacer adaptaciones en relación a la viabilidad empresarial.

3) La Administración Financiera de los Negocios, en Productos y Servicios de cualquier PYME, los empresarios y gerentes deberían aplicar el 1 conocimiento científico y tecnológico, mediante un Modelo de Gestión Estratégico Gerencial. Esto es, aplicar un enfoque empresarial para alcanzar el nivel de productividad y competitividad, que permita obtener la rentabilidad adecuada y el desarrollo de cada PYME y de todo el sector de empresarios del cantón Guayaquil.

\section{Bibliografía}

Chanse, R. Alquilano. Dirección y Administración de la Producción. Editorial Fondo de Cultura Barcelona España. Edición 1997.

Medina, Juan. Programa de Actualización en Venta Profesional. Editorial Cortón Publicación Quito Ecuador. Edición 2004. 
Kaplan, S. Robert y Johnson H. Thomas Johnson. Revelance Lost The Rise and Fail of Management Accounting. Boston, Harvard Business School Press. Edición 1987.

Goldratt, Eliyhau M. La Meta. Editorial McGraw Hill New York EE.UU. Edición 2001.

Porter, Michael. Teoría Cadena de Valor. Editorial McGraw Hill Madrid España. Edición 2001.

Jimenez, Fernando. Administración Estratégica. Editorial Miami Internacional EE.UU. Edición 2005.

Drucker, Peter. Los Desafíos de la Gerencia del Siglo XXI. Editorial NORMA, Bogotá Colombia. Edición 2004.

Sallenave, Paúl. Gerencia y Planeación Estratégica. Editorial Norma Bogotá Colombia. Edición 2002. 